\title{
Anwendung der Resonanzfluoreszenz zur Zink- und Cadmium-Bestimmung in biologischem Material
}

\author{
Von R. KLaus \\ Aus dem Kontroll-Laboratorium der Fa. E. Merck A.G., Darmstadt (Direktor: Prof. Dr. K. G. Krebs)
}

(Eingegangen am 1. Februar 1966)

Es wird ein relativ einfaches Bestimmungsverfahren für die Elemente Zink und Cadmium in verschiedenen biologischen Flüssigkeiten beschrieben. Die Analysen können mit Ausnahme von Cadmium in Humanserum bzw. Rattenurin ohne vorherige Anreicherung durchgeführt werden. Der zusätzliche apparative Aufwand für ein mit einem Flammenphotometer ausgerüstetes Labor ist relativ gering.

A comparatively simple method for the determination of elementary zinc and cadmium in various biological liquids is described. With the exception of cadmium all analyses in human serum and rat urine are possible without prior concentration. For laboratories with a flame photometer, only comparatively little additional equipment is needed.

Die Bestimmung der Elemente Natrium, Kalium und Calcium in biologischem Material wird heute im allgemeinen emissionsflammenphotometrisch durchgeführt. Bei der Anwendung dieses Verfahrens zur MagnesiumAnalyse sind allerdings einige Nachteile unverkennbar. So liegt $z$. B. der Magnesium-Gehalt des Serums in einer Größenordnung, die eine wesentliche Verdünnung zur direkten flammenphotometrischen Bestimmung mit handelsüblichen Geräten nicht zuläßt. Ferner erfordert, da der Zusammenhang zwischen Meßwert und Konzentration für die allein in Frage kommende Analysenlinie $\lambda=285,2 \mathrm{~m} \mu$ nicht linear ist, die Magnesium-Analyse im Gegensatz zur Natrium-, Kalium- und Calcium-Bestimmung unter allen Umständen die Aufnahme einer Eichkurve. Die Lage der Magnesium-Linie im Bereich einer sehr starken Flammeneigenstrahlung dürfte ebenfalls nicht dazu angetan sein, die Methode als besonders geeignet $z u$ bezeichnen, wenn auch das Arbeiten mit einem Doppelmonochromator hier eine gewisse Verbesserung bringt (1).

In den letzten Jahren bot sich nun ein gänzlich neues analytisches Verfahren zur Magnesium-Bestimmung an, die sog. Atomabsorption. Hierbei wird die Absorption eines entsprechenden Hintergrundstrahlers beim Durchtritt durch die Flamme, in welche die Analysenflüssigkeit gesprüht wird, gemessen. Die wesentlichsten Vorteile dieser Methode sind eine größere Empfindlichkeit, die eine Verdünnung z. B. des Serums von 1:20 noch zuläßt, Linearität der Eichkurve in einem relativ großen Konzentrationsbereich, sowie das Ausbleiben einiger Störeffekte in der gasförmigen Phase des Analysengutes, die sich bei der thermischen Anregung nachteilig bemerkbar machen können. Sehr ausführlich berichtet wurde hierüber in einer Arbeit von R. Herrmann (2). Dieses Verfahren läßt sịch nun auf einige weitere Elemente anwenden, deren Analyse seither fast ausschließlich kolorimetrisch erfolgte, u. a. auch auf Zink und Cadmium. Zink und Cadmium, deren Resonanzlinien ähnlich Magnesium im kurzwelligen UV liegen, sind in den meisten Körperflüssigkeiten noch in einer Menge vorhanden, die ausreicht, um eine atomabsorptionsphotometrische Analyse durchführen zu können $(3,4)$.

Eine mit der atomaren Absorption in engstem Zusammenhang stehende Resonanzstrablung ist nun nach neueren
Untersuchungen ebenfalls zur Analyse der Elemente Zink und Cadmium geeignet $(5,6)$. Es zeigt sich hierbei, daß die Nachweisgrenzen, gleiche Versuchsbedingungen vorausgesetzt, gegenüber der atomaren Absorption bei kleineren Werten liegen. Die die Absorptionsmessung charakterisierenden Eigenschaften im Flammenplasma können fast ausnahmslos auf die Fluoreszenzmessung übertragen werden. $\mathrm{Da}$ darüber hinaus der zusätzliche apparative Aufwand geringer ist, unternahmen wir den Versuch, die beiden zur Diskussion stehenden Elemente in verschiedenen biologischen Flüssigkeiten nach der Fluoreszenzmethode zu bestimmen.

\section{Methodik}

MeBanordnung

Die von uns verwendete Meßanordnung setzt das Vorhandensein eines Spektralphotometers voraus.

Abbildung 1 zeigt die wesentlichen Bestandteile im Prinzip, Abbildung 2 ein Zeiss'sches Spektralphotometer PMQ II mit der Zerstäuber-Brenner-Kombination und der Entladungslampe vor dem Eintrittsspalt des Monochromators. Eine Zink- bzw. Cadmium-

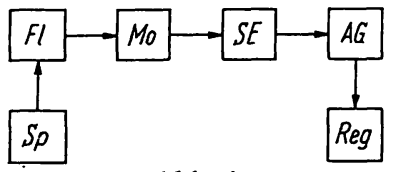

Abb. 1

Blockschaltbild der Meßanordnung

$\mathrm{Fl}=$ Flamme; $\mathrm{Sp}=$ Spektrallampe; Mo = Monochromator; $\mathrm{SE}$ = Strahlungsempfänger; $A G=$ Anzeigegerät; Reg, = Registriergerät

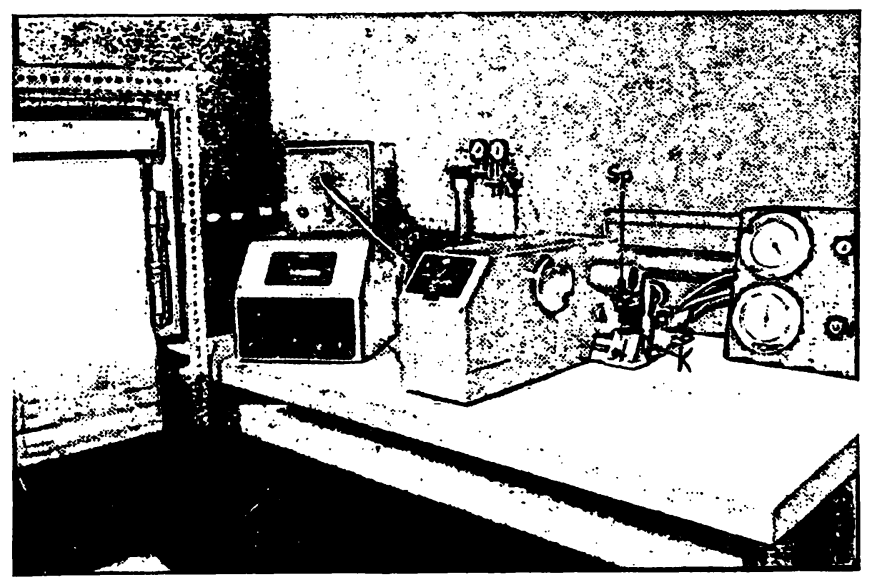

Abb. 2

Gesamtansicht der Meßanordnung 
Osram-Spektrallampe (Sp) bestrahlt unter einem Winkel von etwa $45^{\circ}$ zur optischen Achse den von dem Monochromator abgebildeten Teil der Flamme. Die zu analysierende Flüssigkeit wird durch die Kapillare (K) des Direktzerstäubers in die Flamme gebracht. An dem Anzeigegerät exfolgt die Ablesung des Meßwertes. Nach Anbau eines Wellenlängenantriebes an den Monochromator können in Verbindung mit einem an das Anzeigegerät angeschlossenen Kompensationsschreiber die Meßwerte registriert werden.

Zink-Analyse im Serum

Die quantitative Bestimmung eines Elementes in einem Mehrkomponentensystem wird erfahrungsgemäß in den meisten Fällen durch die einzelnen Bestandteile des Analysengutes mehr oder weniger beeinflußt. Bei den in der Literatur beschriebenen kolorimetrischen Schwermetallanalysen z. B. in biologischem Material umgeht man diese Störeffekte, indem man eine Isolierung des interessierenden Elementes vornimmt. Die eigentliche Ermittlung des Gehaltes erfolgt dann unter Verwendung einer reinen Metall-Eichkurve. Die bei emissionsflammenphotometrischen Alkali- bzw. Erdalkalianalysen auftretenden Störungen werden $u$. a. durch Zugabe von z. T. spezifisch wirkenden Substanzen zu den Meßlösungen ausgeschaltet. Ein weiteres, in der Praxis weniger bekanntes, von uns aber mit gutem Erfolg angewandtes Verfahren basiert auf der Zugabe von steigenden Mengen des zu prüfenden Elementes zur Analysenlösung. Durch Extrapolation der Meßwert-Konzentrations-Kurve erhält man schließlich den gesuchten Gehalt.

Die bei den nachfolgend beschriebenen Zink- und Cadmium-Analysen gebrauchte Methode zur Berücksichtigung von Störeffekten wurde erstmals von Fukushima (7) angewandt, Bei dieser, als Koeffizientenmethode bekannt gewordenen Analyse, wird unter Verwendung einer reinen Metall-Eichkurve ein erster Annäherungswert $\left(\mathrm{C}_{0}\right)$ für die Analysenlösung ermittelt. Der Korrekturfaktor ergibt sich aus der Zugabe einer bestimmten Menge $(\Delta C)$ des zu prüfenden Elementes und dem aus der Eichkurve abgelesenen, korrespondierenden Wert $\left(\Delta C^{\prime}\right)$. Somit errechnet sich der gesuchte Gehalt nach der Gleichung $\mathrm{C}=\mathrm{C}_{0} \cdot \frac{\Delta \mathrm{C}}{\Delta \mathrm{C}^{\prime}}$; Abbildung 3 veranschaulicht den Zusammenhang.

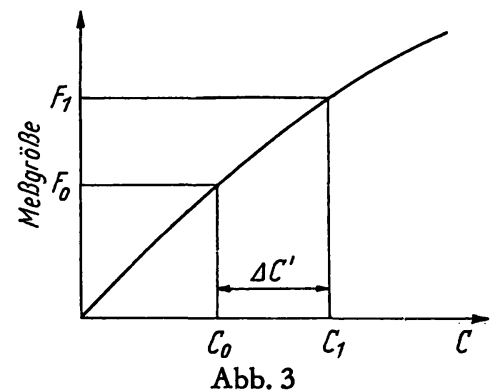

Angenommene Eichkurve zur Zn-Bestimmung nach dem Konzentrationskoeffizientenverfahren

Bei der Festlegung unserer Analysenbedingungen im einzelnen versuchten wir, drei voneinander nicht unabhängige Forderungen zu erfüllen: a) der Substanzverbrauch soll sich auf ein Minimum beschränken. b) die Vergleichslösungen sollen möglichst einfach sein. c) die zu korrigierenden Störungen sollen gering sein. Die für das Verfahren ermittelte Nachweisgrenze erlaubt für die Zink-Bestimmung ein Nachverdünnen des Serums von etwa $1+2$, was sich günstig auf den Substanzverbrauch auswirkt. Zur Prüfung der Punkte b) und c) stellten wir

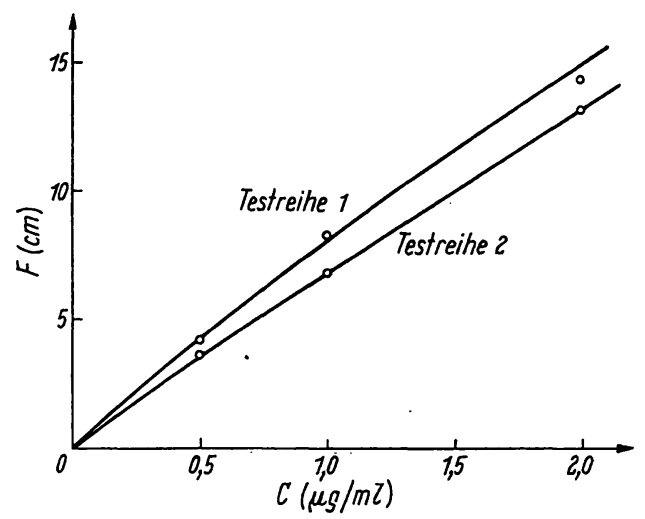

Abb. 4

Abhängigkeit Meßwert $(\mathrm{F})$ - Zinkkonzentration (C)

Testreibe 1: wäßr. Lösungen; Testreibe 2 : Lösungen mit $\mathrm{Na}, \mathrm{K}, \mathrm{Ca}$ und $\mathrm{Mg}$-Zusätzen in der Größe des jeweiligen physiologischen Mittelwertes

$z$ wei Testreihen her, welche je $0,5,1,0$ und $2,0 \mu \mathrm{g} / \mathrm{ml}$ Zink enthielten. Bei Reihe 1 handelte es sich um rein wäßrige Lösungen, Reihe 2 enthielt zusätzlich die .Elemente $\mathrm{Na}, \mathrm{K}, \mathrm{Ca}$ und $\mathrm{Mg}$, zusammengefaßt in einer Lösung „HEL“, in Mengen, die dem jeweiligen physiologischen Mittelwert des Serums entsprechen, ferner zur Angleichung der Oberflächenșpannung ein entsprechendes Netzmittel. Abbildung 4 zeigt die aus den Messungen resultierenden Kurven. Auf der Ordinate ist die von den registrierten Zinkfluoreszenzkurven abgegriffene Peakhöhe aufgetragen, als Abszisse die entsprechende Zinkkonzentration. Die, wenn auch nur geringen Unterschiede zwischen Reihe 1 und Reihe 2, lassen im Sinne der Punkte b) und c) das Arbeiten mit der Lösung „HEL“ als zweckmäßig erscheinen.

Von den im Rahmen der Vorversuche durchgeführten Analysen sei noch der folgende erwähnt. Ein im Handel befindliches Modellserum, welches mehrere organische, sowie die wesentlichsten anorganischen Serum-Komponenten enthält, diente als Ausgangssubstanz für die ZinkAnalyse. Aus der unter Verwendung der bereits erwähnten Lösung „HEL“ hergestellten Eichreihe wurdẹn die für die drei Testlösungen

a) Modellserum

b) Modellserum + Zinkzusatz $\Delta \mathrm{C}_{1}(=0,91 \mu \mathrm{g})$

c) Modellserum + Zinkzusatz $\Delta C_{2}(=1,91 \mu \mathrm{g})$

gültigen Konzentrationen ermittelt. Die nach der Gleichung $\mathrm{C}=\mathrm{C}_{\mathbf{0}} \cdot \frac{\Delta \mathrm{C}}{\Delta \mathrm{C}^{\prime}}$ errechneten Zink-Gehalte des Modellserums betragen im Falle der Kombination a und $\mathrm{b}, \mathrm{C}_{1}=1,9 \mu \mathrm{g} / \mathrm{ml}$, bei Berücksichtigung von $\mathrm{b}$ und $\mathrm{c}$, $\mathrm{C}_{2}=1,8 \mu \mathrm{g} / \mathrm{m} \mathrm{l}$, also zwei Werte, die als gut übereinstimmend bezeichnet werden können.

Bei der anschließend beschriebenen Zink-Analyse eines Humanserums wurde die zur Verfügung stehende Menge geteilt. Während die erste Hälfte einer direkten Analyse 
zugeführt wurde, sollte die zweite Bestimmung nach vorheriger Veraschung durchgeführt werden. Letztere erfolgte nach einem allgemeinen üblichen Verfahren: $5 \mathrm{ml}$ Serum werden mit je $1 \mathrm{ml}$ konz. Schwefelsäure und Salpetersäure mehrmals in einem Kjeldahl-Kölbchen abgeraucht. Nach Uberspülen in eine Abdampfschale wird das Lösungsmittel eingedampft, der Rückstand mit $5 \mathrm{~m} / \mathrm{N}$-Salzsäure aufgenommen. Analog hierzu wird ein Blindversuch vorbereitet.

Tabelle 1 gibt Aufschluß über die verwendeten Meßlösungen einschließlich der Meßwerte und die mit Hilfe der Eichkurve (Abb. 5) ermittelten Konzentrationen. Ergänzend sind in Abbildung 6 die Spektren der Meßlösungen im Bereich der Zink-Resonanzlinie $\lambda=$ $213,8 \mathrm{~m} \mu$ dargestellt.

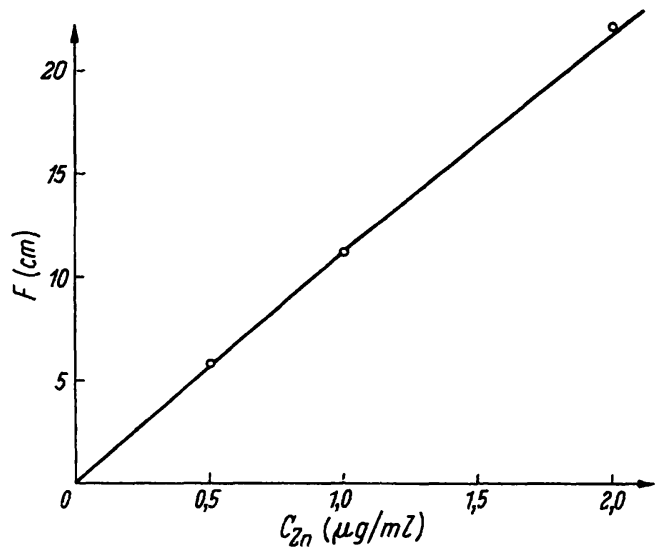

Abb. 5

$\mathrm{Zu}$ Tabelle 1 gehörende Eichkurve

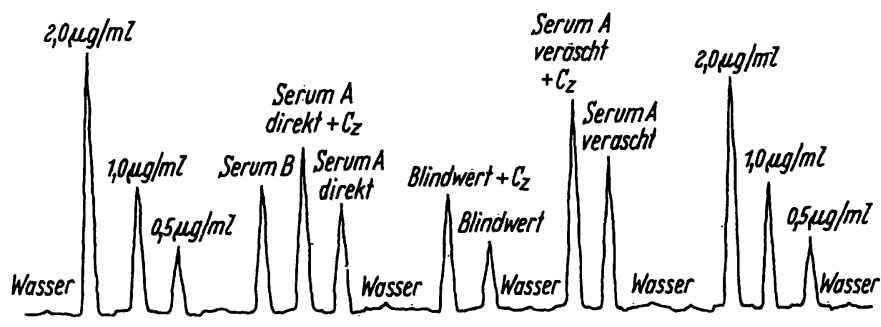

Abb. 6

Registrierkurven im Bereich der Zink-Resonanzlinie für die Meßlösungen dèr Tabelle 1
Eine Gegenüberstellung der beiden Zink-Gehalte führt zu einem befriedigenden Ergebnis:

Zn-Gehalt des Serums $\left\{\begin{array}{c}\text { nach Veraschen gemessen: } \\ 0,8_{3} \mu \mathrm{g} / \mathrm{m} l \\ \text { direkt gemessen: } \\ 0,9_{6} \mu \mathrm{g} / \mathrm{m} l\end{array}\right.$

Um ein besseres Bild von der Brauchbarkeit des Verfahrens zu bekommen, wurden von einem weiteren Serum Mehrfachbestimmungen durchgeführt, von denen hier nur die Ergebnisse wiedergegeben seien ( $\mathrm{Zn}$ Gehalte in $\mu \mathrm{g} / \mathrm{m} l)$ :

$\begin{array}{ll}\text { Serum direkt analysiert } & \left\{\begin{array}{l}\text { graphische Extrapolation: } \\ 1,2 \\ \text { Koeffizienten-Methode } \\ 1,2\end{array}\right. \\ \text { Serum verascht } & \left\{\begin{array}{l}\text { graphische Extrapolation: } \\ 1,5 \\ \text { Koeffizienten-Methode: } \\ 1,4\end{array}\right.\end{array}$

\section{Ergebnisse}

Faßt man sämtliche von uns in diesem Zusammenhang durchgeführten Zink-Analysen in den verschiedenen, nicht pathologischen Seren zu einem Mittelwert zusammen, so erhält man einen mittleren Zink-Gehalt von $1,2 \mu \mathrm{g} / \mathrm{m} l$.

Die in der Biologie übliche Angabe eines Normalbereiches (Mittelwert $\pm 2 \mathrm{~s}$ ) verlangt eine relativ große Anzahl von Analysen. Obwohl diese Bedingung im vorliegenden Fall nicht erfüllt ist, haben wir mit Hilfe der 15 untersuchten Seren die Schranken dieses Normalbereiches errechnet. Sie liegen bei $1,2 \pm 0,4 \mu \mathrm{g} / \mathrm{m} l$. -Demgegenüber steht ein nach neuesten kolor imetrischen Analysen (MALMSTRÖM-Methode) ermittelter Zink-Gehalt im Serum von Gesunden von $116,0 \pm 12,2 \mu \mathrm{g} \%$ (8).

Zink-Analysen in Urin

Mit zwei weiteren biologischen Flüssigkeiten wurde der Anwendungsbereich der Methode untersucht. Der Gang

Tab. 1: Zusammenstellung der für eine Serum-Zink-Analyse verwendeten Meßlösungen einschließlich der Meßwerte bzw. der nach Fukushima korrigierten Werte

\begin{tabular}{|c|c|c|c|c|}
\hline & $\begin{array}{l}\text { Meßwerte } \\
\text { (cm) }\end{array}$ & $\begin{array}{l}\text { F (cm), Meß- } \\
\text { werte abz. } \\
\text { Meßwert } \\
\text { Wasser } \\
\end{array}$ & Konz. $(\mu \mathrm{g} / \mathrm{m} l)$ & \\
\hline $\begin{array}{l}\text { Wasser } \\
0,5 \mu \mathrm{g} \mathrm{Zn} / \mathrm{m} l \\
1,0 \mu \mathrm{g} \mathrm{Zn/m} l \\
2,0 \mu \mathrm{g} \mathrm{Zn/ml}\end{array}$ & $\begin{array}{r}0,3 \\
6,1 \\
11,5 \\
22,5\end{array}$ & $\begin{array}{c}0 \\
5,8 \\
11,2 \\
22,2\end{array}$ & . & \\
\hline $\begin{array}{l}5 \mathrm{~m} l \text { Serum verascht } / \\
5 \mathrm{~m} l_{\mathrm{N}-\mathrm{HCl}} \\
5 \mathrm{~m} l \text { Serum verascht }+ \\
2,5 \mu \mathrm{g} \mathrm{Zn} / 5 \mathrm{~m} l_{\mathrm{N}-\mathrm{HCl}}\end{array}$ & $\begin{array}{l}11,9 \\
18,9\end{array}$ & $\begin{array}{l}13,6 \\
18,6\end{array}$ & $\begin{array}{l}1,2_{2} \\
1,6_{6}\end{array}$ & $\Delta \mathrm{C}^{\prime}=0,4_{4} \mu \mathrm{g} / \mathrm{m} l ; \mathrm{C}=\mathrm{C}_{0} \cdot \frac{\Delta \mathrm{C}}{\Delta \mathrm{C}^{\prime}}=1,2_{2} \cdot \frac{0,5}{0,4_{4}}=1,3_{9} \mu \mathrm{g} / \mathrm{m} l$ \\
\hline $\begin{array}{l}\text { Blindlösung verascht/ } \\
5 \mathrm{~m} l \mathrm{~N}-\mathrm{HCl} \\
\text { Blindlösung verascht }+ \\
2,5 \mu \mathrm{g} \mathrm{Zn} / 5 \mathrm{~m} / \mathrm{N}-\mathrm{HCl}\end{array}$ & $\begin{array}{r}5,9 \\
10,9\end{array}$ & $\begin{array}{r}5,6 \\
10,6\end{array}$ & $\begin{array}{l}0,5_{0} \\
0,9_{5}\end{array}$ & $\Delta C^{\prime}=0,4_{5} \mu \mathrm{g} / \mathrm{ml} ; C=C_{0} \cdot \frac{\Delta C}{\Delta C^{\prime}}=0,5_{0} \cdot \frac{0,5}{0,4_{5}}=0,5_{6} \mu \mathrm{g} / \mathrm{m} l$ \\
\hline $\begin{array}{l}\text { Serum (direkt) } \\
\text { Serum }+2,5 \mu \mathrm{g} \mathrm{Zn} / 5 \mathrm{~m} l\end{array}$ & $\begin{array}{r}9,6 \\
14,4 \\
\end{array}$ & $\begin{array}{r}9,3 \\
14,1 \\
\end{array}$ & $\begin{array}{l}0,8_{3} \\
1,2_{6} \\
\end{array}$ & $\Delta \mathrm{C}^{\prime}=0,4_{3} \mu \mathrm{g} / \mathrm{ml} ; \mathrm{C}=\mathrm{C}_{0} \cdot \frac{\Delta \mathrm{C}}{\Delta \mathrm{C}^{\prime}}=0,8_{4} \cdot \frac{0,5}{0,4_{3}}=0,9_{8} \mu \mathrm{g} / \mathrm{m} l$ \\
\hline
\end{tabular}


der Analysen entspricht dem der Serum-Zink-Analysen. Wir beschränken uns daher auf die Angabe eines aus einer relativ kleinen Anzahl von Einzelwerten ermittelten arbitrarisch begrenzten Bereiches.

Mittlerer Zink-Gehalt von Humanurin einschließlich der max. ermittelten Abweichung $1,0 \pm 0,1 \mu \mathrm{g} / \mathrm{m} l$

Mittlerer Zink-Gehalt von Rattenurin einschließlich der max. ermittelten Abweichung 0,20 $\pm 0,04 \mu \mathrm{g} / \mathrm{m} l$.

\section{Cadmium-Analysen}

Wenn auch Cadmium-Analysen in biologischem Material in physiologischer Hinsicht nicht die Bedeutung der Zink-Bestimmungen haben, so soll trotzdem auf die Möglichkeit der Bestimmung mit Hilfe der Resonanzfluoreszenz hingewiesen werden.

Die Zink-Meßanordnung wird lediglich dadurch abgeändert, daß die Zink-Spektrallampe durch eine entsprechende Cadmium-Lampe ersetzt wird. Zur Messung eignet sich die Resonanzlinie bei $\lambda=228,8 \mathrm{~m} \mu$.

Die ersten mit Seren durchgeführten Versuche ließen erkennen, daß eine direkte Cadmium-Analyse ohne Anreicherung in vielen Fällen nur die Angabe eines Grenzwertes zuläßt. Dieser beträgt unter Zugrundelegung der Definition von KaISER und Specker (9) $0,1 \mu \mathrm{g} / \mathrm{ml}$. Um dennoch eine effektive Aussage machen zu können, wurde der Weg der Anreicherung beschritten.

$7 \mathrm{~m} l$ des Analysengutes wurden nach den oben gemachten Angaben verascht. Der Rückstand wurde mit $2 \mathrm{~m} / \mathrm{N}$-Salzsäure aufgenommen. Analog hierzu wurde ebenfalls ein Blindversuch vorbereitet.

Tabelle 2 zeigt eine Zusammenstellung der verwendeten Meßlösungen einschließlich der Meßwerte und der aus der Eichkurve (Abb. 7) ermittelten Konzentrationen. Schließlich sind in Abbildung 8 die registrierten Spektren der Meßlösungen im Bereich der Cadmium-Resonanzlinie wiedergegeben. Die Analyse ergab für das untersuchte Serum einen Cadmium-Gehalt von $0,04_{3} \mu \mathrm{g} / \mathrm{ml}$. Drei weitere Seren, die aufgrund ihrer

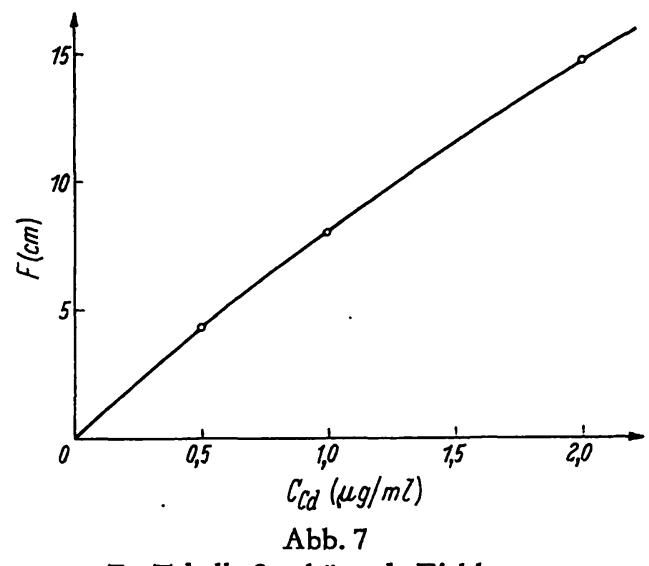

Zu Tabelle 2 gehörende Eichkurve

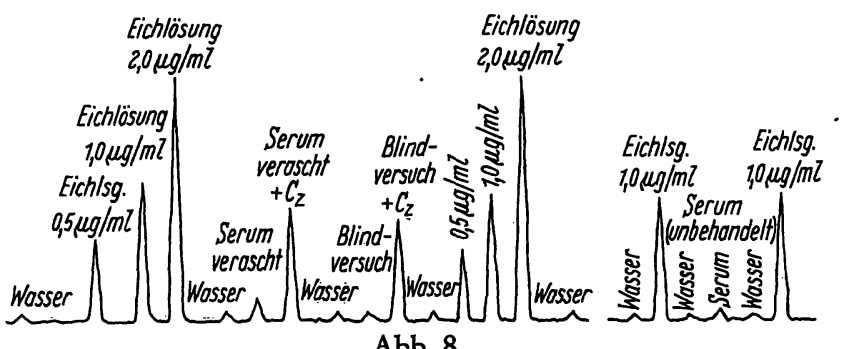

Registrierkurven im Bereich der Cd-Resonanzlinie für die Meßlösungen der Tabelle 2

etwas höheren Cadmium-Gehalte obne Anreicherung analysiert wurden, lieferten Werte im Bereich der Nachweisgrenze. Allerdings muß bei dieser Bestimmung mit einem relativ großen Analysenfehler gerechnet werden.

Nicht unerwähnt sei die Möglichkeit der Cadmium-Bestimmung in Urinen. Während uns für Human-Urin eine Veraschung mit anschließender Anreicherung nicht unbedingt erforderlich erscheint - es wurde ein Gehalt von $0,18 \mu \mathrm{g} / \mathrm{m} l$ gefunden - dürfte man $z$. B. bei Rattenurin bei Cadmium-Gehalten von etwa $0,03 \mu \mathrm{g} / \mathrm{m} l$ auf diese angewiesen sein.

Tab. 2 : Zusammenstellung der für eine Serum-Cadmium-Analyse verwendeten Meßlösungen einschließlich der Meßwerte bzw. der nach Fukushrma korrigierten Werte

\begin{tabular}{|c|c|c|c|}
\hline & $\begin{array}{l}\text { Meßwerte } \\
\quad(\mathrm{cm})\end{array}$ & $\begin{array}{c}\text { F (cm), Meß- } \\
\text { werte abz. } \\
\text { Meßwert } \\
\text { Wasser }\end{array}$ & Konz. $(\mu \mathrm{g} / \mathrm{m} l)$ \\
\hline $\begin{array}{l}\text { Wasser } \\
0,5 \mu \mathrm{g} \mathrm{Cd} / \mathrm{m} l \\
1,0 \mu \mathrm{g} \mathrm{Cd} / \mathrm{m} l \\
2,0 \mu \mathrm{g} \mathrm{Cd} / \mathrm{m} l\end{array}$ & $\begin{array}{r}0,4 \\
4,7 \\
8,3 \\
15,1\end{array}$ & $\begin{array}{r}0 \\
4,3 \\
7,9 \\
14,7\end{array}$ & . \\
\hline $\begin{array}{l}7 \mathrm{~m} / \text { Serum verascht } \\
2 \mathrm{~m} / \mathrm{N}-\mathrm{HCl}(\mathrm{A}) \\
1 \mathrm{~m} / \mathrm{LgA}+1 \mu \mathrm{g} \mathrm{Cd}\end{array}$ & $\begin{array}{l}1,4 \\
7,0\end{array}$ & $\begin{array}{l}1,0 \\
6,6\end{array}$ & $\left.\begin{array}{l}0,1_{1} \\
0,8_{1}\end{array}\right\} \Delta \mathrm{C}^{\prime}=0,7_{0} \mu \mathrm{g} / \mathrm{m} l, \mathrm{C}=\mathrm{C}_{0} \frac{\Delta \mathrm{C}}{\Delta \mathrm{C}^{\prime}}=0,11 \cdot \frac{1}{0,7_{0}}=0,15 \mu \mathrm{g} / \mathrm{ml}$ \\
\hline $\begin{array}{l}\text { Blindlösung verascht/ } \\
2 \mathrm{~m} / \mathrm{N}-\mathrm{HCl}\end{array}$ & 0,4 & 0 & $=0$ \\
\hline
\end{tabular}

\section{Literatur}

1. Prugger, H., Zeiss-Informationen 53, 95 (1964). - 2. HerrMANN, R., Z. ges. exp. Lud. 135, 569 (1962). - 3. SLAvin, W. und S. Sprague, Fa. Perkin-Elmer, Tips für Atomabsorption Nr. 17, 1 (1963). - 4. Willis, J. B., Analytic. Chem. 34, 614 (1952). - 5. Winneforder, I. B., Analytic. Chem. 36, 161, 165 (1964). - 6.
KLAUS, R., Zeiss-Mitt (Stuttgart) 4, H. 1 (1966). - 7. Furushrma, SH. u. a., Mikrochim. Acta (Wien), 183 (1957). - 8. KRASNoschischekow, S., Med. Klinik 25, 2332 (1965). - 9. KAISER, H. und H. SPECKER, Z. analyt. Chem. 149, 46 (1956).

R. Klaus, Fa. E. Merck AG, 61 Darmstadt, Frankfurter Str. 250 